- This study is a comprehensive cost-effectiveness analysis of advanced conscious sedation techniques in primary care versus dental general anaesthetic in hospital, for children requiring dental treatment.

- It is shown that the sedation techniques can be a cost-effective alternative to hospital general anaesthetic.

- The ability to implement these techniques safely and cost-effectively in primary care depends on specially trained staff and dedicated facilities with a high throughput of patients.

- The research further informs the debate about the best way to manage patients requiring such interventions.

\title{
A comparison of the 'cost per child treated' at a primary care-based sedation referral service, compared to a general anaesthetic in hospital
}

\author{
K. Jameson, ${ }^{1}$ P. A. Averley, ${ }^{2}$ P. Shackley ${ }^{3}$ and J. Steele ${ }^{4}$
}

Aim To compare the cost-effectiveness of dental sedation techniques used in the treatment of children, focusing on hospital-based dental general anaesthetic (DGA) and advanced conscious sedation in a controlled primary care environment.

Methods Data on fees, costs and treatment pathways were obtained from a primary care clinic specialising in advanced sedation techniques. For the hospital-based DGA cohort, data were gathered from hospital trusts in the same area. Comparison was via an average cost per child treated and subsequent sensitivity analysis.

Results Analysing records spanning one year, the average cost per child treated via advanced conscious sedation was $£ 245.47$. As some treatments fail (3.5\% of cases attempted), and the technique is not deemed suitable for all patients (4-5\%), DGA is still required and has been factored into this cost. DGA has an average cost per case treated of $€ 359.91,46.6 \%$ more expensive than advanced conscious sedation. These cost savings were robust to plausible variation in all parameters. Conclusion The costs of advanced conscious sedation techniques, applied in a controlled primary care environment, are substantially lower than the equivalent costs of hospital-based DGA, informing the debate about the optimum way of managing this patient group.

${ }_{1}^{*}$ Department of Economics and Related Studies, University of York; ${ }^{2}$ Principal Dentist, Queensway Anxiety Management Clinic, 170 Queensway, Billingham, Teesside, TS23 2NT; ${ }^{3}$ Senior Lecturer, Institute of Health and Society, Newcastle University, 21 Claremont Place, Newcastle upon Tyne, NE2 4AA; ${ }^{4}$ Clinical Professor/Consultant, School of Dental Sciences, Newcastle University, Framlington Place, Newcastle upon Tyne, NE2 4BW

${ }^{*}$ Correspondence to: Mr Kevin Jameson, c/o P. Averley, Queensway Dental Practice and Anxiety Management Clinic, 170 Queensway, Billingham, Teesside, TS23 2NT Email:kevin_jameson@hotmail.com

Online article number E13

Refereed Paper - accepted 15 November 2006

DOI: $10.1038 /$ bdj.2007.631

${ }^{\circledR}$ British Dental Journal 2007; 203: E13

\section{INTRODUCTION}

Two groups of children pose particularly difficult management problems for the dentist; those who are extremely anxious and unable to cope with behavioural management, and those who require an invasive and/or extensive procedure. The conventional way to treat these patients has been to use the conscious sedation technique of relative analgesia (RA), with inhaled nitrous oxide as a sedative. In the event that RA is ineffective, or unavailable, this will usually result in referral for dental general anaesthetic (DGA).

In the United Kingdom and elsewhere, current interpretation of government guidelines ${ }^{1}$ forbids the use of DGA, unless performed in a hospital. The definition of conscious sedation which is accepted within the $\mathrm{UK}^{1-4}$ is: 'a technique in which the use of a drug or drugs produces a state of depression of the central nervous system enabling treatment to be carried out, but during which verbal contact with the patient is maintained throughout the period of sedation. The drugs and techniques used to provide conscious sedation for dental treatment should carry a margin of safety wide enough to render loss of consciousness unlikely.' Historically, RA has been the mainstay for this sedation method for children. Recently, however, advanced conscious sedation techniques - which use a combination of inhaled $40 \%$ nitrous oxide in oxygen, with up to $0.3 \%$ sevoflurane and intravenous agents such as midazolam and fentanyl - have been used in highly controlled environments and shown to be clinically effective. ${ }^{5}$

Since October 1998, Queensway Anxiety Management Clinic in the North East of England has managed referred anxious patients via RA and, when necessary, more advanced conscious sedation techniques in a highly controlled environment supported by appropriately trained and experienced staff. This clinic is in an area of relative deprivation where demand for DGA was amongst the highest in the country, and the 
Table 1 Numbers on the community dental service waiting lists

\begin{tabular}{|l|l|l|l|}
\hline & North Tees & South Tees & Total \\
\hline $\begin{array}{l}\text { Referral rate (patients } \\
\text { per week) }\end{array}$ & $10-12$ & $20-30$ & - \\
\hline Awaiting assessment & 86 & 365 & 451 \\
\hline $\begin{array}{l}\text { Assessed and awaiting } \\
\text { short DGA }\end{array}$ & 66 & 78 & 144 \\
\hline $\begin{array}{l}\text { Assessed and awaiting } \\
\text { long DGA }\end{array}$ & 85 & 39 & 124 \\
\hline Total & 237 & 482 & 719 \\
\hline
\end{tabular}

formation of a primary care-based unit, to provide a service for anxious patients, was partly as a response intended to ease the pressure on local hospitals. The treatment is provided with all the efficiencies of a dental practice, but with appropriate specialist teams, training and facilities. Elsewhere there is, by necessity, still a heavy reliance on DGA. Freeman and Carson ${ }^{6}$ found that only 29\% of the general dental practitioners they investigated had RA equipment available. This finding lies in stark contrast to recommendations that 'sedation be used in preference to general anaesthesia wherever possible.'

In the United Kingdom, DGAs are generally provided by the community dental service in a local hospital. However, with other calls on beds and theatre time there is the potential for waiting lists for DGA to be considerable. Such waiting times may affect the child's quality of life and may also exacerbate the child's underlying anxiety. ${ }^{7}$ Anxiety management that can be provided in primary care may have the potential to reduce waiting times, but could also have additional benefits. Arch et $a l .{ }^{8}$ found that children who choose to undergo RA 'will become more familiar with the dental environment', leading to a less phobic child, which in turn may also reduce future dependence on DGA. ${ }^{9}$ There may also be benefits in terms of patient and parent satisfaction. Blain and Hill ${ }^{10}$ found that whilst $71 \%$ of RA patients' parents rated their child as being happy with the treatment, this was the case in only 33\% of the DGA cohort, and $86 \%$ said they would opt for the same treatment if their child required future extractions, compared to only $24 \%$ of DGA parents. ${ }^{10}$ Conscious sedation techniques may also hold an advantage inasmuch as that they allow for the patient to have their surgery completed over multiple visits, as opposed to a single session - a feature which the resource constraints of DGA will typically require.

In summary, there is evidence that sedation techniques hold advantages for the patients and their families over DGA in terms of satisfaction, quality of life and the ability to provide appropriate clinical dentistry. The safety benefits are also implicit, provided the techniques are used by trained and experienced teams with appropriate facilities. This is an essential pre-requisite of this type of management. The efficacy of techniques that reduce the need for DGA have been evaluated in a clinical trial and have been shown to be highly effective. $^{5}$ One of the final determinants of the utility of alternatives to DGA will be economic. This paper aims to assess the
Table 2 Estimated waiting time at current throughput (months)

\begin{tabular}{|l|l|l|}
\hline \multirow{2}{*}{} & \multicolumn{2}{|c}{ Waiting Times (months) } \\
\cline { 2 - 3 } & North Tees & South Tees \\
\hline Awaiting assessment & $5-6$ & 10 \\
\hline $\begin{array}{l}\text { Assessed and awaiting } \\
\text { short DGA }\end{array}$ & $5-6$ & 4 \\
\hline $\begin{array}{l}\text { Assessed and awaiting } \\
\text { long DGA }\end{array}$ & 14 & 5 \\
\hline \begin{tabular}{l} 
Total \\
\hline
\end{tabular} & 15.78 & 14.33 \\
\hline
\end{tabular}

cost-effectiveness of new methods of advanced conscious sedation (combination techniques of intravenous and inhalation agents) used in a highly controlled primary care environment, compared with hospital-based DGA services.

No differences in oral health outcomes are incorporated into this analysis; such aspects are analysed in greater depth elsewhere. ${ }^{5}$ This study forms part of a wider series, including qualitative research to assess the attitudes of children and adults.

\section{METHODS}

Data collection: hospital service providing DGA

Data were collected from the North Tees, South Tees and Hartlepool regions in the North East of England, the same area as the specialist primary care sedation service. Cost data were obtained on relevant 'healthcare resource group' (HRG) charges. ${ }^{11}$ These represent the average cost of performing a particular procedure - calculated over the entire National Health Service (NHS) - and is the sum paid to the supplier of care to cover the complete treatment episode, from initial assessment to discharge. It was deemed unlikely that the HRG charge would accurately represent the actual cost incurred by the community dental service to perform a DGA. However, a recent audit has estimated the cost of providing a DGA in this context in the area concerned and these data were also obtained. The latter (audit data) are likely be more accurate, but the former are also considered for purposes of generalisability to areas without specialist clinics.

Data on the trusts' waiting list profiles and patient throughput were collected to estimate the time DGA patients can expect to wait for treatment. This was compared to the waiting time for patients in the specialist clinic over the same period.

\section{Data collection: primary care service providing advanced conscious sedation techniques}

Data comprised information gathered routinely during the patient's appointment and subsequent administration. Data drawn from the computerised database included the number of treatment failures using advanced conscious sedation and the details of onward referral - for a hospital DGA, or back to their general dental practitioner. Along with establishing treatment pathways, the system also made it possible to calculate the fees claimed for each patient from the NHS. 
In order to bring the number of cases within manageable limits, a random sample of patients was chosen. Four weeks were chosen by random number allocation, one from each quarter of the year (September 29th 2003, December 1st 2003, February 1st 2004 and June 14th 2004). The number of children treated and the number of visits required to complete treatment were established. If treatment had not been completed at the time of study, the child was excluded from the analysis.

Treatment costs, including costs for fillings and extractions were established. To this, a single assessment fee was added, along with the appropriate number of sedation fees. The assessment and sedation fees are those paid to the primary care-based service by the NHS and do not alter with the sedation technique employed.

\section{Data analysis}

Data were used to estimate the 'average cost per child treated' for DGA and for advanced conscious sedation techniques. For the latter, the cost of treatment failures and referrals were added to the basic treatment cost. This additional cost is calculated by multiplying the DGA-based cost by the probability of referring a child for DGA after assessment, and the probability of referral after treatment failure under advanced sedation.

Results were subjected to a rigorous sensitivity analysis, where input parameters and values were uni-dimensionally altered. By varying one parameter at a time (through an extendedly-plausible range), the robustness of the results could be analysed. The four parameters coming under such scrutiny were: hospital service costs, the probability of treatment failure and the probability of referral for a DGA, both after assessment at the primary care-based service and following treatment failure.

\section{RESULTS}

\section{Hospital service providing DGA}

\section{Waiting lists}

Data on the local area waiting lists (from the health trusts in each area) are shown in Table 1. Those categorised as a short DGA case require simple extractions, whilst those listed for a long DGA require intubation and/or more lengthy procedures. These figures include some children with special needs that may make them inappropriate for treatment in an acute care environment, but give an indication of proportions of children needing different types of services in the study area. Mean age is not available, as access to patient records was not possible.

The community dental service had been allocated two afternoon sessions (33/4 hour each) per week to treat their patients. Either eight short or three long DGAs were possible in any one session. The patients in Table 1 can, therefore, expect to wait the times shown in Table 2.

\section{Cost per child treated}

Using HRG charges, a DGA is classified as either category 1 or category 2 oral surgery, entailing a cost of $£ 568$ or $£ 616$, respectively. ${ }^{11}$ Assuming that these represent short and long DGAs, a weighted-average HRG cost is: $((£ 568 \times 144)+(£ 616$ $\mathrm{X} 124)) \div(144+124)=£ 590.21$ (where the numbers awaiting treatment in Table 1 are used to weight the data - 144 and 124 awaiting a short and long DGA, respectively).

An alternative method to HRG charges, for the assessment of a cost per child treated, was made available via a recent audit of the local community dental service. Using these data and excluding dental staff costs, a short and long DGA were estimated to cost $£ 172$ and $£ 459$ per procedure, respectively. Costs included the salary of non-dental staff (anaesthetists and administration staff, including during the assessment stage), consumables, equipment and portering costs. At each session, one senior dental officer and two dental nurses were present, commanding a total salary cost of $£ 187$ per session. Thus it can be estimated that, once dental staff costs are included, each short DGA procedure costs $£ 195.38(£ 172+(£ 187 \div 8)=£ 195.38)$ and each long DGA procedure costs $£ 521.33(£ 459+(187 \div 3)=£ 521.33)$. Weighting these costs by the figures in Table 1 to take into account the general proportions of short and long cases, gives an average DGA cost of $((£ 195.38 \times 144)+(£ 521.33 \times 124)) \div$ $(144+124)=£ 346.19$.

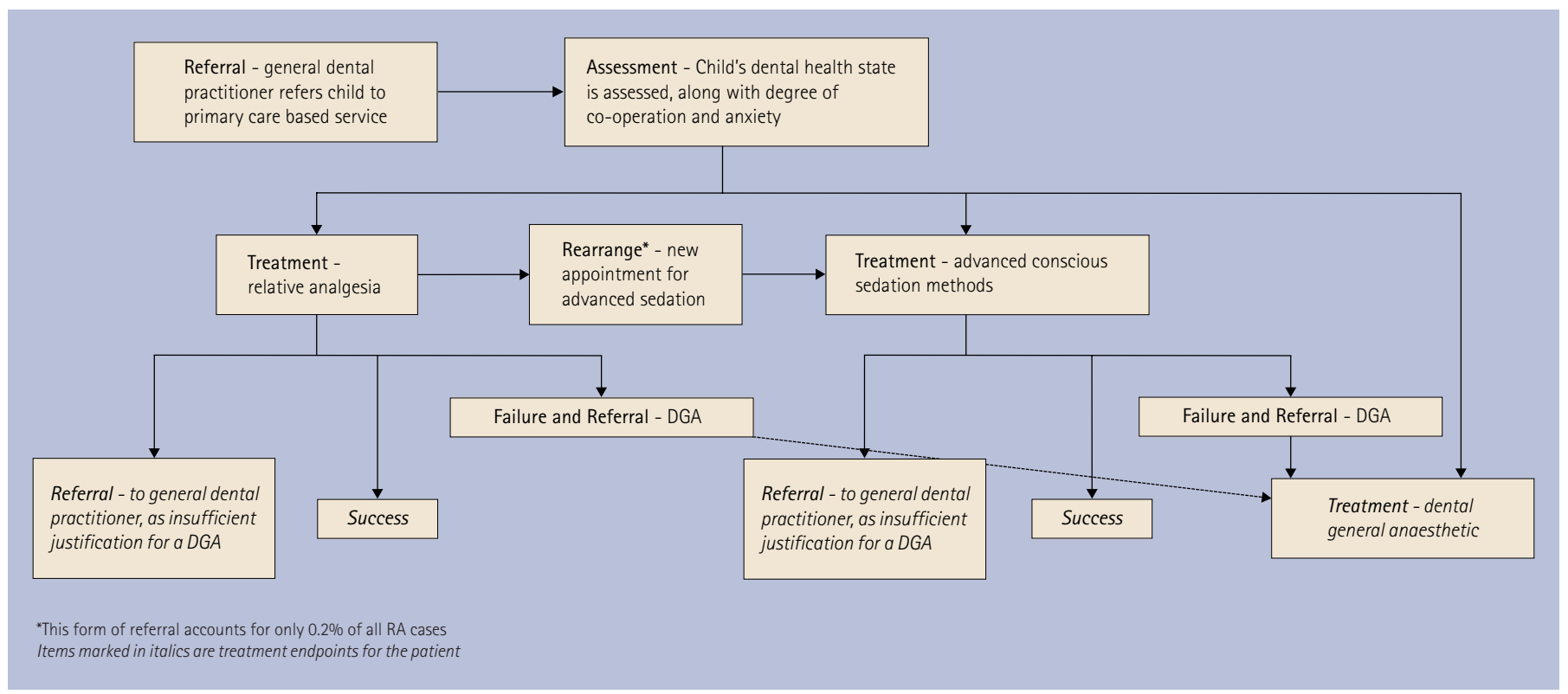

Fig. 1 Treatment pathways for a patient at the primary care-based service 


\begin{tabular}{|c|c|c|}
\hline \multirow{2}{*}{ Procedure } & \multicolumn{2}{|l|}{ Fee } \\
\hline & Pre $1 / 5 / 04$ & Post $1 / 5 / 04$ \\
\hline Assessment & $€ 29.52$ & $€ 30.38$ \\
\hline RA & $€ 71.05$ & $€ 73.11$ \\
\hline Failed RA & $£ 23.63$ & $£ 24.32$ \\
\hline Advanced conscious sedation & $€ 79.36$ & $£ 81.66$ \\
\hline
\end{tabular}

Additionally, an in-patient day unit with two beds is reserved in case of complications with the DGA, although only one bed has been used at any one time in the last two years. Appropriate data are available to allow us to estimate bed costs, ${ }^{12}$ the cost per bed day lying between $£ 39$ and $£ 118$. For the purposes of this analysis, the lower value was used, implying an additional cost of $£ 8,112$ per year ( $£ 39 \times 2$ beds per session $\mathrm{x} 2$ sessions per week $\mathrm{x} 52$ weeks per year). The need to keep beds available is, therefore, associated with an additional mean cost of $£ 13.72$ per DGA performed, leading to an average cost per-case treated via DGA of $£ 359.91$. This is $64 \%$ cheaper than the cost suggested by analysis through HRGs, ${ }^{11}$ but is calculated at a local level, taking into account the availability of specialist services.

\section{Primary care service providing advanced conscious sedation techniques \\ Waiting lists}

Through an analysis of the waiting lists at the primary carebased service, along with the protocols in operation, it was found that children requiring non-urgent treatment would wait around four weeks to be assessed, followed by a further four weeks to receive their treatment (on average, eight weeks in total). In contrast, urgent cases (those referred with severe pain and/or sepsis) would be assessed within 48 hours, and receive treatment within 72 hours. In all cases, the time to assessment would be from the initial referral by the child's general dental practitioner.

In the treatment period, 3,291 patients were treated, of whom 2,771 were children. All patients seen required a prior assessment visit. No child appeared in more than one week of the four which were analysed.

\section{Treatment pathways}

Based on the child's degree of co-operation, anxiety and the invasiveness of the intended procedure, they will embark on one of three treatment pathways; simple RA, advanced conscious sedation, or referral for a DGA (Fig. 1). An audit of referrals at the primary care-based service identified that $4-5 \%$ of patients would be referred for DGA at the assessment stage.

In cases where RA fails (1.5\% of all attempts), a patient may be referred for a DGA, to their general dental practitioner (if there is insufficient justification for a DGA), or given a new clinic appointment, where treatment will be attempted via advanced conscious sedation (which accounts for only $0.2 \%$ of all RAs). Only when the patient began to receive treatment via advanced conscious sedation were they included for analysis in this study; therefore, costs associated with RA are not included.
Children failing to cope with treatment under advanced conscious sedation will either be referred for a DGA, or back to their general dental practitioner. Of the 2,771 children undergoing advanced conscious sedation, 97 (3.5\%) failed to cope with treatment. Forty-two of these patients $(1.52 \%$ of all patients and $43.3 \%$ of all failures) were referred to the hospital service for a DGA and 55 (1.98\% and 56.7\%, respectively) were referred back to their general dental practitioner, as there was insufficient justification for a DGA.

The maximum waiting time for a patient referred to the specialist clinic was two months.

\section{Treatment cost}

Applicable fees from the NHS are shown in Table 3, which increased on May 1st 2004.

Using the cost and patient list data supplied by the specialist practice, the mean age of a patient seen in the four randomly chosen weeks was 9.78 years old $(\mathrm{SD}=3.52)$ and required 1.27 visits ( $S D=0.55)$ to complete treatment $(n=205)$. Taking into account the increase in fees and the typical number of visits, the average cost of assessment and sedation was $£ 131.18$. Combining this with the average cost of dental treatment for this group of children, calculated from the statement of dental remuneration (£92.60), the average treatment cost per patient for the advanced sedation methods was £223.78. However, other aspects of the process in the primary care environment must be taken into account.

Some patients, 4-5\% (assume 4.5\%), will be immediately referred for a DGA and must be accounted for. A further 1.52\% of patients were referred for a DGA after treatment failure via advanced conscious sedation. The additional cost of these referrals can be calculated by multiplying the cost of a DGA by the sum of these two probabilities, ie £359.91 x $(0.045+$ $0.0152)=£ 21.69$.

Combining the basic cost of treatment under advanced conscious sedation with the additional cost incurred through the referral of patients for a DGA leads to an average cost per child treated of $£ 245.47(£ 223.78+£ 21.69)$.

\section{Cost-effectiveness}

The cost of the advanced conscious sedation techniques is $£ 114.44$ lower than that of DGA ( $£ 344.74$ lower if HRG costs are used $\left.^{11}\right)$. Given the assumption of no difference in oral health outcome between the techniques, this translates into an additional cost of $46.6 \%$ (140.44\%) when a child is treated via DGA as opposed to advanced conscious sedation. Due to the nature of the data employed and the concomitant uncertainty, these results must be subjected to a sensitivity analysis.

\section{Sensitivity analysis}

The probability of DGA referral following treatment failure (currently 1.52\%) was found to have no effect on the optimal treatment choice.

\section{Hospital costs}

Due to the possible inaccuracies in data obtained on treatment cost, it was important to calculate the price at which DGA becomes the more cost-effective option. Figure 2 illustrates how the costs of DGA and advanced conscious sedation change as the cost of DGA is multiplied by a cost-reducing 


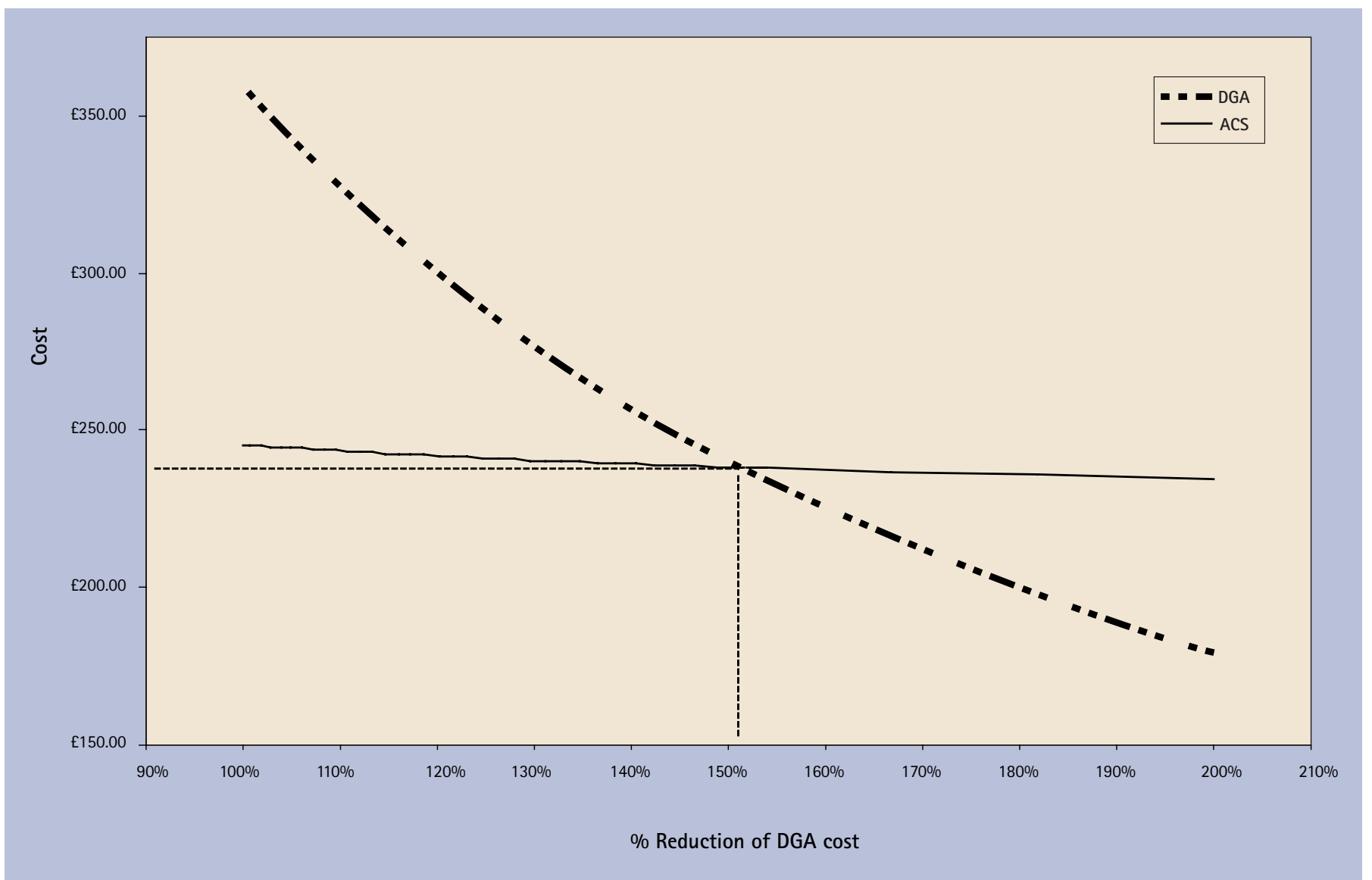

Fig. 2 Sensitivity analysis of hospital costs for dental general anaesthetic (DGA) against advanced conscious sedation (ACS) costs

percentage. The horizontal axis shows the factor that the cost of DGA is divided by; for example, at 200\%, the cost of DGA is divided by 2.0 .

Although not as sensitive as DGA, it can be seen that the cost of the advanced techniques does change with the cost of DGA. This is due to the implicit link between the two, via referrals.

It was found that when the reduction factor reaches 151.14\%, DGA becomes the more cost effective option at $£ 238.12$ versus $£ 238.13$, ie DGA would need to be 51\% cheaper than its current cost to become cost effective. This percentage differs from the $46.6 \%$ discussed above, as it takes into account how the cost of advanced conscious sedation is linked to the cost of DGA through patient referrals. Due to the relatively high degree of accuracy offered by the recent audit which was used to calculate costs, this reduction would seem unlikely.

\section{Probability of treatment failing}

With all other data inputs remaining at their base case levels, the failure rate of the advanced sedation techniques was varied between 0 and 100\%. It was estimated that the failure rate must reach 77\% (compared to the current level of 3.5\%) for DGA to become the more cost-effective option. However, if the failure rate was so high, the method would almost certainly be abandoned.

\section{Probability of referral following assessment}

The total cost of a patient referred for a DGA (after assessment in the primary care practice) is the sum of the assessment cost in the practice (Table 3 ) and the full cost of a DGA. The DGA will become more cost-effective than the advanced methods if a suitably high proportion of patients from the primary care centre are referred for a DGA. Analyses determined that this point would be reached if $36.32 \%$ of all those assessed were referred on to the hospital.

Whilst there is no reason to preclude such a situation from arising, it does seem extreme considering the present rate of 4-5\%. It is, of course, dependent on the characteristics of patients presenting.

\section{DISCUSSION}

This investigation has demonstrated that treatment via advanced sedation techniques, where the child is conscious and responsive throughout, is more cost-effective compared to treatment via DGA. Using 'average cost per child treated' as the outcome measure, base case analysis found the average cost per patient treated under advanced sedation techniques and DGA to be $£ 245.47$ and $£ 359$.91, respectively - a ratio nearing $1: 1 \frac{1 / 2}{2}$. Sensitivity analysis showed results were robust to plausible variation in all the parameters and inputs involved.

Previous studies in this area ${ }^{7,10,13}$ have produced similar results to those found here, though none were as detailed and complete in their analysis. Blain and Hill ${ }^{10}$ found that within the out-patient service of a UK dental hospital, the overall cost ratio of RA, a simple inhalation sedation technique, to

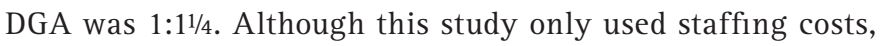
once the cost of consumables and other factors are taken into account, a ratio closer to that noted above may be established. According to their study, both parental and patient opinion favoured RA. ${ }^{10}$ This report's findings also tie in with those of Shaw et al. ${ }^{13}$ where RA was 30\% cheaper than a day-case DGA 
and 57\% cheaper than an in-patient DGA. ${ }^{13}$ Once again, with the authors only considering salary costs, these results may be a close match to those established here.

Through the inclusion of costs in addition to salary costs alone, this study can be regarded as the most comprehensive cost-effectiveness analysis of DGA and its nearest alternatives. Previous work has considered only RA, but there is a significant difference in the level of a child's co-operation achievable through RA, compared with that through advanced conscious sedation. This is evidenced by the small number of children whose treatment failed with the latter. In clinical terms, advanced sedation techniques are a viable alternative to DGA where RA fails, or cannot be used. They must be delivered by appropriately trained and experienced teams with appropriate facilities; nevertheless, this study is therefore considering two real alternatives: DGA and advanced conscious sedation techniques.

As with all previous studies, the analysis reported here does not incorporate a quality of life assessment. Further work would be beneficial to make an additional assessment of the impacts of both techniques on quality of life. Utility-based oral health related quality of life measures are available, ${ }^{14}$ as are child-specific measures, ${ }^{15}$ whilst a contingent valuation exercise or conjoint analysis could also be applied. Not only would this allow for the capture of patient preferences, but also the ability to incorporate the impact of longer waiting times and possibly the clinical outcome. ${ }^{5,16}$ Advanced sedation techniques have characteristics and clinical outcomes that would normally be expected to favour the patient, whether this is the shorter waiting time, implied lower levels of anxiety, ${ }^{10-12}$ or treatment plans with better long-term oral health outcomes. ${ }^{5,16}$ It seems likely that richer data such as this would strengthen, rather than weaken, the case for making advanced sedation services available.

In order to obtain a balanced picture, we should consider what is missing from the data employed. Within the hospital data, it was not known how many patients had special needs. Such patients would not be treated in a primary care setting, thus creating a systematic difference between the cohorts, and perhaps maintaining the mean cost for hospital DGA at a higher level. Conversely, with the option of advanced services, it is possible that a smaller proportion would receive a DGA. Considering the small proportion of patients in the primary care environment that were truly deemed to require a DGA (at worst, around 6\%), it is unlikely that the $86 \%$ of children receiving a DGA in the hospital-based service (14\% were managed via RA - data not shown) had a special need severe enough to justify its use. Nevertheless this is a relevant consideration. The mean age of children treated in the hospital sector was also not available.

Using a relatively straightforward outcome measure, this analysis has shown that advanced sedation techniques are more cost-effective than DGA. Dental general anaesthetic would need to be $46 \%$ cheaper than its current cost to become cost-effective. These cost differences were robust to changes in parameter values, only altering in magnitude.
Implicitly captured within the fees paid to the primary carebased service are the capital and sunk costs of establishing the clinic, including staff training. As the clinic has a high level of patient throughput, it is able to spread these set-up costs across a wide patient pool and the reimbursement fees paid to the clinic are sufficient to cover such costs. These economies of scale should be borne in mind when considering establishing such a relatively specialised service. The clinic in this study lies in an area of high need, justifying a substantial service; similar services on a much smaller scale may not be as efficient.

This study is part of a wider picture and is intended to inform the debate about the best ways of delivering high quality dental care to anxious children; in future it should be possible to include even richer outcome measures. The clinical benefits of these sedation techniques have already been demonstrated in a clinical trial setting. ${ }^{5}$ The long-term hope is for an evolutionary approach to service development and review, allowing the wider uptake of increasingly effective, safe, cost-effective and acceptable ways of managing dental anxiety in children.

At the time of conducting this analysis, Kevin Jameson was an MSc student at the University of York. This publication evolved from the dissertation, which was conducted at Newcastle University under his supervisors Phil Shackley and Paul Averley.

1. Department of Health. A conscious decision. A review of the use of general anaesthesia and sedation in primary dental care. London: Department of Health, 2000.

2. Society for the Advancement of Anaesthesia in Dentistry. Standards in conscious sedation for dentistry. Report of an independent expert working group. London: Society for the Advancement of Anaesthesia in Dentistry, 2000.

3. Standing Dental Advisory Committee. Conscious sedation in the provision of dental care. Report of an expert group on sedation for dentistry. London: Department of Health, 2003.

4. The Dental Sedation Teachers Group. Training in conscious sedation for dentistry. The Dental Sedation Teachers Group, 2005. http://www.dstg.co.uk/teaching/

5. Averley P A, Girdler N M, Bond S, Steen N, Steele J. A randomised controlled trial of paediatric conscious sedation for dental treatment using intravenous midazolam combined with nitrous oxide or nitrous oxide/sevoflurane. Anaesthesia 2004; 59: 844-852.

6. Freeman R, Carson P. Relative analgesia and general dental practitioners: attitudes and intentions to provide conscious sedation for paediatric dental extractions. Int J Paediatr Dent 2003; 13: 320-326.

7. Milnes A R. Intravenous procedural sedation: an alternative to general anaesthesia in the treatment of early childhood caries. J Can Dent Assoc 2003; 69: 298-302.

8. Arch L M, Humphris G M, Lee G TR. Children choosing between general anaesthesia or inhalation sedation for dental extractions: the effect on dental anxiety. Int J Paediatr Dent 2001; 11: 41-48.

9. Bryan R A E. The success of inhalation sedation for comprehensive dental care within the Community Dental Service. Int J Paediatr Dent 2002; 12: 410-414.

10. Blain K M, Hill F J. The use of inhalation sedation and local anaesthesia as an alternative to general anaesthesia for dental extractions in children. BrDent J 1998; 184: 608-611.

11. Department of Health. NHS reference costs 2003. London: Department of Health, 2003.

12. Netten A, Curtis L. Unit costs of health and social care. Canterbury: Personal Social Services Research Unit, 2003.

13. Shaw A J, Meechan J G, Kilpatrick N M, Welbury R R. The use of inhalation sedation and local anaesthesia instead of general anaesthesia for extractions and minor oral surgery in children: a prospective study. Int J Paediatr Dent 1996; 6: 7-11.

14. McGrath C, Bedi R. An evaluation of a new measure of oral health related quality of life - OHQoL-UK(W). Community Dent Health 2001; 18: 138-143.

15. Humphris G, Freeman R, Gibson B, Simpson K, Whelton H. Oral health-related quality of life for 8-10 year old children: an assessment of a new measure. Community Dent Oral Epidemiol 2005; 33: 326-332.

16. Harrison M, Nutting L. Repeat general anaesthesia for paediatric dentistry. Br Dent J 2000; 189: 37-39. 